\title{
Spherically Symmetric Gravitational Collapse
}

\author{
M. Sharif *and Khadija Iqbal \\ Department of Mathematics, University of the Punjab, \\ Quaid-e-Azam Campus, Lahore-54590, Pakistan.
}

\begin{abstract}
In this paper, we discuss gravitational collapse of spherically symmetric spacetimes. We derive a general formalism by taking two arbitrary spherically symmetric spacetimes with $g_{00}=1$. Israel's junction conditions are used to develop this formalism. The formulae for extrinsic curvature tensor are obtained. The general form of the surface energy-momentum tensor depending on extrinsic curvature tensor components is derived. This leads us to the surface energy density and the tangential pressure. The formalism is applied to two known spherically symmetric spacetimes. The results obtained show the regions for the collapse and expansion of the shell.
\end{abstract}

Keywords: Gravitational collapse.

\section{Introduction}

It has been an interesting problem to investigate whether the spacetime singularities can be observed or not. Penrose [1] suggested a cosmic censorship conjecture to investigate this problem. This conjecture ensures that end state of a gravitational collapse must be a black hole. There is no theoretical or mathematical proof available to this conjecture. It would be worthwhile to investigate this issue for complete understanding of gravitational collapse or

*msharif@math.pu.edu.pk 
to obtain its correct form. This can be pursued by taking the appropriate geometry of exterior and interior regions and proper junction conditions which allow the matching of these two regions.

Many people [2-4] have discussed gravitational collapse by taking appropriate geometry of interior and exterior regions. Oppenheimer and Synder [5] were the pioneer to study the gravitational collapse. They worked on dust collapse by taking static Schwarzschild in the exterior and Friedmann like solution in the interior spacetime. The conclusion was that the end state of gravitational collapse leads to the formation of a black hole. Misner and Sharp [6] worked on the gravitational collapse by taking a static exterior and a perfect fluid in the interior. Markovic and Shapiro [7] generalized the pioneer's work in the presence of a positive cosmological constant. Lake [8] extended this work by taking both positive and negative cosmological constants. Cissoko et al. [9] studied explicitly the effects of a positive cosmological constant on gravitational dust collapse. He found that there are two physical horizons instead of one and also discussed the effects of cosmological constant on gravitational collapse. Debnath et al. [10] studied quasi-spherical collapse with a positive cosmological constant. They also discussed the apparent horizons and their physical significance. Sharif and Ahmad [11,12] studied gravitational collapse of a perfect fluid with a positive cosmological constant by taking static exterior and non-static interior spacetimes. The same authors generalized this work to five and higher dimensional spacetimes $[13,14]$.

In 1966, Israel $[15,16]$ presented junction conditions by taking interior and exterior spacetimes. A lot of work on gravitational collapse has been done by using these conditions [17-20]. Villas da Rocha et al. [21] studied the self-similar gravitational collapse of perfect fluid using Israel's method. He concludes that when collapse has continuous self-similarity, black hole formation starts with zero mass and when the collapse has no self-similarity, black hole formation starts with a finite non-zero mass. By the same procedure, Pereira and Wang [22,23] discussed the gravitational collapse of cylindrical shells made of counter rotating dust particles. Recently, Sharif and Ahmad [24] generalized this work to plane symmetric spacetime. They derived general formulae for arbitrary plane symmetric spacetimes and then applied this formalism to known plane symmetric spacetimes. These studies have provided some interesting results about gravitational collapse. However, no attempt has been made for spherically symmetric spacetimes.

In this paper, we extend this work to spherical symmetric spacetimes 
with matching conditions. First of all, we develop a formalism for general spherical symmetric spacetimes and then the results are found by taking particular examples. The scheme of this paper is as follows: In section $\mathbf{2}$, we provide the derivation of general formalism by considering two arbitrary spherically symmetric spacetimes with $g_{00}=1$. In sections $\mathbf{3}$ and $\mathbf{4}$, we shall implement these formulae to discuss gravitational collapse by taking two physical spherically symmetric spacetimes. The last section will provide summary and discussion of the results obtained.

\section{General Formalism}

We consider a time-like $3 D$ hypersurface $\Sigma$ which divides a $4 D$ spherically symmetric spacetime into two regions named as interior and exterior spacetimes. For the interior region denoted by $V^{-}$, we take the line element in the form as

$$
d s_{-}^{2}=d t^{2}-X^{-}(t, r) d r^{2}-Y^{-}(t, r)\left(d \theta^{2}+\sin ^{2} \theta d \phi^{2}\right),
$$

where $\left\{\chi^{-\mu}\right\} \equiv\{t, r, \theta, \phi\}$ are the usual spherical polar coordinates. The line element for the exterior region $V^{+}$is given by

$$
d s_{+}^{2}=d T^{2}-X^{+}(T, R) d R^{2}-Y^{+}(T, R)\left(d \theta^{2}+\sin ^{2} \theta d \phi^{2}\right),
$$

where $\left\{\chi^{+\mu}\right\} \equiv\{T, R, \theta, \phi\}$ is another set of spherical polar coordinates.

According to junction condition $[15,16,25,26]$, we assume that the interior and exterior spacetimes are the same on the hypersurface $\Sigma$ which can be expressed as

$$
\left(d s^{2}\right)=\left(d s_{-}^{2}\right)_{\Sigma}=\left(d s_{+}^{2}\right)_{\Sigma} .
$$

The equations of hypersurface $\Sigma$ in the coordinates $\chi^{ \pm \mu}$ are written as

$$
\begin{gathered}
f_{-}(r, t)=r-r_{0}(t)=0, \\
f_{+}(R, T)=R-R_{0}(T)=0 .
\end{gathered}
$$

Using Eqs.(4) and (5), the interior and exterior metrics reduce to the following form respectively

$$
\left(d s_{-}^{2}\right)_{\Sigma}=\left[1-X^{-}\left(t, r_{0}(t)\right) r_{0}^{2}(t)\right] d t^{2}-Y^{-}\left(t, r_{0}(t)\right)\left(d \theta^{2}+\sin ^{2} \theta d \phi^{2}\right),
$$




$$
\left(d s_{+}^{2}\right)_{\Sigma}=\left[1-X^{+}\left(T, R_{0}(T)\right){R_{0}^{\prime}}^{2}(T)\right] d T^{2}-Y^{+}\left(T, R_{0}(T)\right)\left(d \theta^{2}+\sin ^{2} \theta d \phi^{2}\right)
$$

where prime denotes the ordinary differentiation with respect to the indicated argument. The metric on the hypersurface is written as

$$
\left(d s^{2}\right)_{\Sigma}=\gamma_{a b} d \xi^{a} d \xi^{b}=d \tau^{2}-Y(\tau)\left(d \theta^{2}+\sin ^{2} d \phi^{2}\right) .
$$

Here $\left\{\xi^{a}\right\}=\{\tau, \theta, \phi\},(a=0,2,3)$ are the coordinates of the hypersurface $\Sigma$ and $\tau$ denotes the proper time of the surface. Making use of Eqs.(6)-(8) into the junction condition (3), it follows that

$$
\begin{gathered}
d \tau=\left[1-X^{-}\left(t, r_{o}(t)\right){r_{0}^{\prime}}^{2}(t)\right]^{\frac{1}{2}} d t=\left[1-X^{+}\left(T, R_{0}(T)\right){R_{0}^{\prime}}^{2}(T)\right]^{\frac{1}{2}} d T \\
Y(\tau)=Y^{-}\left(t, r_{o}(t)\right)=Y^{+}\left(T, R_{o}(T)\right) .
\end{gathered}
$$

From Eqs.(4) and (5), the outward unit normals $n_{\mu}^{ \pm}$to the hypersurface $\Sigma$ in the coordinates $\chi^{ \pm}$can be evaluated as

$$
\begin{gathered}
n_{\mu}^{-}=\left[\frac{X^{-}}{\left[1-X^{-}{r_{0}^{\prime}}^{2}(t)\right.}\right]^{\frac{1}{2}}\left(-r_{0}^{\prime}(t), 1,0,0\right), \\
n_{\mu}^{+}=\left[\frac{X^{+}}{\left[1-X^{+} R_{0}^{\prime 2}(T)\right]}\right]^{\frac{1}{2}}\left(-R_{0}^{\prime}(T), 1,0,0\right) .
\end{gathered}
$$

The extrinsic curvature tensor $K_{a b}^{ \pm}$on the hypersurface $\Sigma$ is defined as $[22,23]$

$$
K_{a b}^{ \pm}=n_{\sigma}^{ \pm}\left(\frac{\partial^{2} x_{ \pm}^{\sigma}}{\partial \varepsilon^{a} \partial \varepsilon^{b}}+\Gamma_{\mu \nu}^{\sigma} \frac{\partial x_{ \pm}^{\mu}}{\partial \varepsilon^{a}} \frac{\partial x_{ \pm}^{\nu}}{\partial \varepsilon^{b}}\right), \quad(\sigma, \mu, \nu=0,1,2,3), \quad(a, b=0,2,3) .
$$

From this expression, the non-vanishing components of $K_{a b}^{+}$for exterior spacetime are given by

$$
\begin{aligned}
K_{\tau \tau}^{+} & =\frac{\left(X^{+}\right)^{\frac{1}{2}}}{\left[1-X^{+} R_{0}^{\prime 2}(T)\right]^{\frac{3}{2}}}\left[R_{0}^{\prime \prime}(T)+\frac{X^{+}{ }_{, T}}{X^{+}} R_{0}^{\prime}(T)\right. \\
& \left.+\frac{X^{+}{ }_{, R}}{2 X^{+}} R_{0}^{\prime 2}(T)-\frac{X^{+}{ }_{, T}}{2} R_{0}^{\prime 3}(T)\right], \\
K_{\theta \theta}^{+} & =\frac{1}{2}\left[\frac{X^{+}}{\left[1-X^{+} R_{0}^{\prime 2}(T)\right]^{\frac{1}{2}}}\left[-\frac{Y^{+}{ }_{, R}}{X^{+}}-Y^{+}{ }_{, T} R_{0}^{\prime}(T)\right]\right. \\
K_{\phi \phi}^{+} & =\sin ^{2} \theta K_{\theta \theta}^{+} .
\end{aligned}
$$


The non-vanishing components of $K_{a b}^{-}$for interior spacetime can be obtained from the above expressions by making the replacement

$$
X^{+}, Y^{+}, R_{0}(T), T, R \rightarrow X^{-}, Y^{-}, r_{0}(t), t, r .
$$

In terms of $K_{a b}^{ \pm}$and $\gamma_{a b}$, the surface energy-momentum tensor is defined as $[15,16,22,23]$

$$
S_{a b}=\frac{1}{\kappa}\left\{\left[K_{a b}\right]-\gamma_{a b}[K]\right\},
$$

where $\kappa$ is the coupling constant and

$$
\left[K_{a b}\right]=K_{a b}^{+}-K_{a b}^{-}, \quad[K]=\gamma^{a b}\left[K_{a b}\right] .
$$

Using Eq.(14) and the corresponding expressions for $K_{a b}^{-}$, we can write $S_{a b}$ in the form

$$
S_{a b}=\rho \omega_{a} \omega_{b}+p\left(\theta_{a} \theta_{b}+\phi_{a} \phi_{b}\right), \quad(a, b=\tau, \theta, \phi),
$$

where $\rho$ is the surface energy density, $p$ is the tangential pressure provided that they satisfy some energy conditions [27] and $\omega_{a}, \theta_{a}, \phi_{a}$ are unit vectors defined on the surface given by

$$
\omega_{a}=\delta_{a}^{\tau}, \quad \theta_{a}=Y^{\frac{1}{2}} \delta_{a}^{\theta}, \quad \phi_{a}=Y^{\frac{1}{2}} \sin \theta \delta_{a}^{\phi} .
$$

Here $\rho$ and $p$ are given by

$$
\rho=\frac{2}{\kappa Y}\left[K_{\theta \theta}\right], \quad p=\frac{1}{\kappa}\left\{\left[K_{\tau \tau}\right]-\frac{\left[K_{\theta \theta}\right]}{Y}\right\} .
$$

\section{Application}

In this section, we apply the general formalism derived in the previous section by considering two known spherically symmetric spacetimes to discuss the gravitational collapse. For the interior region, we take Minkowski spacetime given by

$$
d s_{-}^{2}=d t^{2}-d r^{2}-r^{2}\left(d \theta^{2}+\sin ^{2} \theta d \phi^{2}\right) .
$$

For the exterior region, the metric is taken as [28]

$$
d s_{+}^{2}=d T^{2}-d R^{2}-a^{2}(T-R)\left(d \theta^{2}+\sin ^{2} \theta d \phi^{2}\right),
$$


where $a$ is an arbitrary function of $T-R$. We re-write the above solution by taking $\epsilon \equiv T-R$ for the sake of simplicity as

$$
d s_{+}^{2}=d T^{2}-d R^{2}-a^{2}(\epsilon)\left(d \theta^{2}+\sin ^{2} \theta d \phi^{2}\right) .
$$

Using Eqs.(21) and (23), the junction conditions (9) and (10) take the form

$$
d \tau=\left[1-R_{0}^{\prime 2}(T)\right]^{\frac{1}{2}} d T=\left[1-r_{0}^{\prime 2}(t)\right]^{\frac{1}{2}} d t,
$$

and

$$
r_{0}(t)=a\left(\epsilon_{0}\right),
$$

where $\epsilon_{0}=T-R_{0}(T)$. From Eqs.(24) and (25), we have

$$
\left(\frac{d T}{d t}\right)^{2}=\frac{1}{\Delta^{2}} \equiv \frac{1}{\left[\left(1-R_{0}^{\prime 2}(T)\right)+{a^{\prime}}^{2}\left(\epsilon_{0}\right)\left(1-R_{0}^{\prime}(T)\right)^{2}\right]},
$$

where

$$
\Delta=\sqrt{\left[\left(1-R_{0}^{\prime 2}(T)\right)+{a^{\prime 2}}^{2}\left(\epsilon_{0}\right)\left(1-R_{0}^{\prime}(T)\right)^{2}\right]} .
$$

From Eqs.(25) and (26), we get

$$
\begin{aligned}
r_{0}^{\prime \prime}(t) & =\frac{a^{\prime \prime}\left(\epsilon_{0}\right)\left(1-{R_{0}^{\prime}}^{2}(T)\right)^{2}-a^{\prime}\left(\epsilon_{0}\right) R_{0}^{\prime \prime}(T)}{\left[\left(1-R_{0}^{\prime 2}(T)\right)+a^{\prime 2}\left(\epsilon_{0}\right)\left(1-R_{0}^{\prime}(T)\right)^{2}\right]} \\
& -\frac{1}{\left[\left(1-{R_{0}^{\prime}}^{2}(T)\right)+a^{\prime 2}\left(\epsilon_{0}\right)\left(1-R_{0}^{\prime}(T)\right)^{2}\right]^{\frac{3}{2}}}\left[a^{\prime 2}\left(\epsilon_{0}\right) a^{\prime \prime}\left(\epsilon_{0}\right)\left(1-R_{0}^{\prime}(T)\right)^{4}\right. \\
& \left.-a^{\prime}\left(\epsilon_{0}\right) R_{0}^{\prime}(T) R_{0}^{\prime \prime}(T)\left(1-R_{0}^{\prime}(T)\right)-a^{\prime 3}\left(\epsilon_{0}\right) R_{0}^{\prime \prime}(T)\left(1-R_{0}^{\prime}(T)\right)^{2}\right] .
\end{aligned}
$$

Making use of Eq.(14) and the corresponding expression for $K_{a b}^{-}$into Eq.(20) and considering Eqs.(25)-(28), the expressions for $\rho$ and $p$ take the form

$$
\begin{aligned}
\rho= & \frac{2}{\kappa a\left(\epsilon_{0}\right)\left(1-R_{0}^{\prime 2}(T)\right)^{\frac{1}{2}}}\left[a^{\prime}\left(\epsilon_{0}\right)\left(\left(1-R_{0}^{\prime}(T)\right)+\Delta\right]\right. \\
p= & \frac{1}{\kappa a\left(\epsilon_{0}\right) \Delta\left(1-R_{0}^{\prime}(T)\right)^{\frac{3}{2}}}\left[a\left(\epsilon_{0}\right) \Delta R_{0}^{\prime \prime}(T)-a\left(\epsilon_{0}\right) a^{\prime \prime}\left(\epsilon_{0}\right)\left(1-{R_{0}^{\prime}}^{2}(T)\right)\right. \\
& \left(1-R_{0}^{\prime}(T)\right)^{2}+a\left(\epsilon_{0}\right) a^{\prime}\left(\epsilon_{0}\right) R_{0}^{\prime \prime}(T)\left(1-{R_{0}^{\prime}}^{2}(T)\right)-a\left(\epsilon_{0}\right) a^{\prime}\left(\epsilon_{0}\right) \\
& R_{0}^{\prime}(T) R_{0}^{\prime \prime}(T)\left(1-R_{0}^{\prime}(T)\right)-2 a^{\prime}\left(\epsilon_{0}\right) \Delta\left(1-R_{0}^{\prime 2}(T)\right)\left(\left(1-R_{0}^{\prime}(T)\right)\right. \\
& \left.+\Delta)-2 \Delta^{2}\left(1-R_{0}^{\prime 2}(T)\right)\right]
\end{aligned}
$$


To see the minimum effects of shell on the collapse, we take $p=0$ in Eq.(30), it follows that

$$
\begin{aligned}
& R_{0}^{\prime \prime}(T)=\left(1-{R_{0}^{\prime}}^{2}(T)\right) \\
& \times \frac{\left[a\left(\epsilon_{0}\right) a^{\prime \prime}\left(\epsilon_{0}\right)\left(1-R_{0}^{\prime}(T)\right)^{2}+2 a^{\prime}\left(\epsilon_{0}\right) \Delta\left(1-R_{0}^{\prime}(T)\right)+2 \Delta^{2}\right]}{\left[a\left(\epsilon_{0}\right) \Delta-a\left(\epsilon_{0}\right) a^{\prime}\left(\epsilon_{0}\right) R_{0}^{\prime}(T)\left(1-R_{0}^{\prime}(T)\right)+a\left(\epsilon_{0}\right) a^{\prime}\left(\epsilon_{0}\right)\left(1-{R_{0}^{\prime}}^{2}(T)\right)\right]} .
\end{aligned}
$$

To investigate the spherically symmetric gravitational collapse, we need to solve the above equation for $R_{0}(T)$. However, it is much more complicated to solve this in general to get any insight. Therefore, we consider a particular case in which

$$
R_{0}^{\prime \prime}(T)=\left(1-R_{0}^{2}(T) \beta,\right.
$$

where $\beta$ is assumed to be an arbitrary constant given by

$$
\beta=\frac{\left[a\left(\epsilon_{0}\right) a^{\prime}\left(\epsilon_{0}\right)^{\prime}\left(1-R_{0}^{\prime}(T)\right)^{2}+2 a^{\prime}\left(\epsilon_{0}\right) \Delta\left(1-R_{0}^{\prime}(T)\right)+2 \Delta^{2}\right]}{\left[a\left(\epsilon_{0}\right) \Delta-a\left(\epsilon_{0}\right) a^{\prime}\left(\epsilon_{0}\right) R_{0}^{\prime}(T)\left(1-R_{0}^{\prime}(T)\right)+a\left(\epsilon_{0}\right) a^{\prime}\left(\epsilon_{0}\right)\left(1-R_{0}^{\prime 2}(T)\right)\right]} .
$$

Now integrating Eq.(32), we obtain

$$
R_{0}^{\prime}(T)=\frac{e^{2 \beta T}+e^{2 c_{1}}}{e^{2 \beta T}-e^{2 c_{1}}}
$$

where $c_{1}$ is constant of integration. Here, we consider the following two cases:

$$
\text { (I) } \beta>0 \quad(I I) \quad \beta<0 .
$$

\subsection{Case I}

In this case, we take $\beta=1$ and $c_{1}=0$ for simplicity, then Eq. (34) takes the form

$$
R_{0}^{\prime}(T)=\frac{e^{2 T}+1}{e^{2 T}-1}
$$

Integration of the above equation yields

$$
R_{0}(T)=-T+c_{2}+\ln \left(-1+e^{2 T}\right)
$$




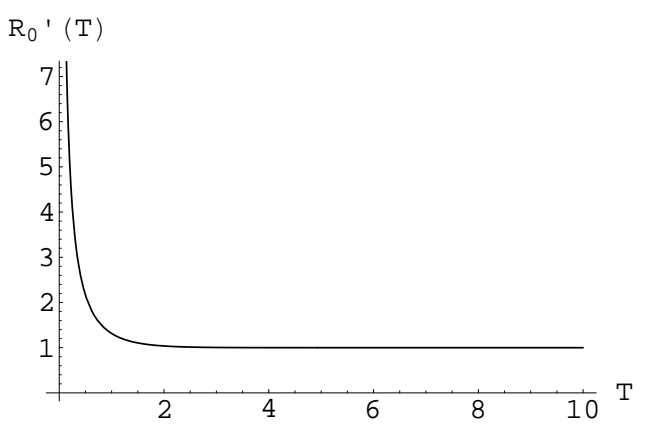

Figure 1: velocity-time graph for $c_{1}=0$.

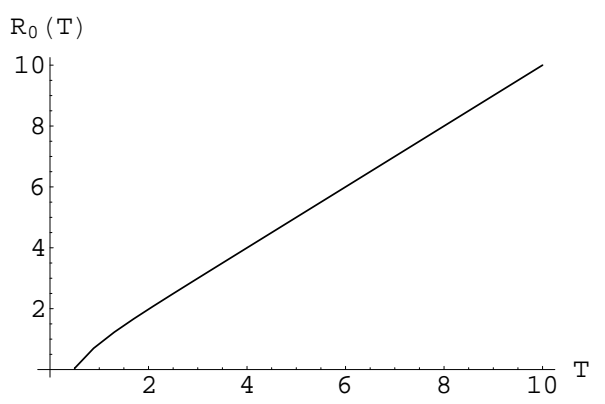

Figure 2: displacement-time graph for $c_{2}=0$.

where $c_{2}$ is also a constant of integration. For $c_{2}=0$, Eqs. (36) and (37) yield respectively



$$
\begin{aligned}
& R_{0}^{\prime}(T)= \begin{cases}2.16359 & , T=0.5 \\
1 & , T=+\infty .\end{cases}
\end{aligned}
$$

This case gives expanding process. It can be seen from figures 1 and 2 that radial velocity is positive and radius increases from some finite value to infinity. The expansion starts from some finite value at some finite time with finite positive velocity and ends at $T=+\infty$, where radial velocity is unity. It is also observed that by considering different values of $c_{2}$ we get the same expanding process. We can take different values of $c_{2}$. The difference for possibilities of $c_{2}$ is that by increasing the values of $c_{2}$, the time interval for the expanding process increases. 


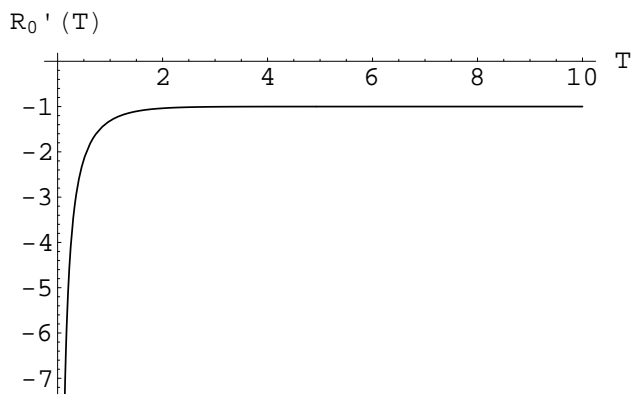

Figure 3: velocity-time graph for $c_{1}=0$.

\subsection{Case II}

Here we take $\beta=-1$ and $c_{1}=0$ for simplicity, then Eq.(34) implies that

$$
R_{0}^{\prime}(T)=\frac{e^{-2 T}+1}{e^{-2 T}-1}
$$

Integration of this equation gives

$$
R_{0}(T)=T+c_{3}-\ln \left(-1+e^{2 T}\right),
$$

where $c_{3}$ is a constant of integration. For $c_{3}=0$, Eqs.(40) and (41) give

$$
\begin{aligned}
& R_{0}(T)= \begin{cases}+\infty & , T=0 \\
0.000026 & , T=0.4812,\end{cases} \\
& R_{0}^{\prime}(T)= \begin{cases}-\infty & , \quad T=0 \\
-1.45678, & T=0.4812 .\end{cases}
\end{aligned}
$$

This case represents a collapsing process. It can be seen from figures 3 and 4 that radial velocity is negative and radius positively decreases from infinity to some finite value. The shell starts collapsing from some finite time with infinite negative velocity and ends with some finite radial velocity at some finite time. It is also found that different values of $c_{3}$ shows the same collapsing process. The difference between possibilities of $c_{3}$ is that by increasing the values of $c_{3}$, the time interval for collapsing process increases. 




Figure 4: displacement-time graph for $c_{3}=0$.

\section{Summary and Discussion}

This paper is devoted to the study of spherically symmetric gravitational collapse using Israel's method. We have taken two arbitrary spherically symmetric spacetimes with $g_{00}=1$. We have developed a general formalism for extrinsic curvature and surface energy-momentum tensor in terms of the metric coefficients and their first derivatives by using Israel's junction condition. As an application of this formalism, we have taken Minkowski spacetime as an interior region whereas a non-static spacetime is taken as an exterior region. The surface energy density and the tangential pressure have been found. In order to see the minimum effects of the shell on the collapse, we have taken the tangential pressure $p=0$.

There arise two main cases, i.e., Case I and Case II. In the Case I, we have taken an arbitrary constant $\beta>0$ and for simplicity its value as unity. Here, we get an expanding process with positive radial velocity and positively increasing radius. The expansion starts from some finite value at some finite time with finite positive velocity. The expansion ends at $T=+\infty$, where radial velocity is unity. It is also observed that time interval for radial velocity decreases with the increase of the constant used in Eq.(34). In the nutshell, we can say that we get an expanding sphere for all values of arbitrary constant $\beta>0$ and the integration constants.

For the Case II, we have taken $\beta<0$ and in particular $\beta=-1$ for simplicity. This case represents a collapsing process with negative radial velocity and radius decreases positively from infinity to some finite value. The shell starts collapsing from some finite time with infinite negative velocity and ends with some finite radial velocity at some finite time. Results also show that by increasing the value of the constant used in Eq.(34), the time 
interval for the radial velocity also increases. Thus it turns out that the case II represents a collapsing sphere for all values of arbitrary and integration constants. It is interesting to mention here that in the cases where collapse occurs, the energy density remains finite. Thus the collapse does not end as a singularity. It would be interesting if this formalism is developed without taking $g_{00}=1$ so that more general examples could be discussed.

\section{Acknowledgment}

We would like to thank Mr. Zahid Ahmad for the fruitful discussions on the subject.

\section{References}

[1] Penrose, R.: Riv. Nuovo Cimento 1(1969)252.

[2] Ghosh, S.G. and Deshkar, D.W.: Int. J. Mod. Phys. D12(2003)317.

[3] Ghosh, S.G. and Deshkar, D.W.: Gravitation and Cosmology 6(2000)1.

[4] Debnath, U., Nath, S. and Chakraborty, S.: Mon. Not. R. Astron. Soc. $\mathbf{3 6 9}(2006) 1961$.

[5] Oppenheimer, J.R. and Synder, H.: Phys. Rev. 56(1939)455.

[6] Misner, C.W. and Sharp, D.: Phys. Rev. 136(1964)B571.

[7] Markovic, D. and Shapiro, S.L.: Phys. Rev. D61(2000)084029.

[8] Lake, K.: Phys. Rev. D62(2000)027301.

[9] Cissoko, M., Fabris, J.C., Gariel, J., Denmat, G.L. and Santos, N.O.: arXiv:gr-qc/9809057.

[10] Debnath, U., Nath, S. and Chakraborty, S.: Gen. Relativ. Grav. D37(2005)215.

[11] Sharif, M. and Ahmad, Z.: Mod. Phys. Lett. A22(2007)1493. 
[12] Sharif, M. and Ahmad, Z.: Mod. Phys. Lett. A22(2007)2947.

[13] Sharif, M. and Ahmad, Z.: J. Korean. Phys. Soc. 52(2008)980.

[14] Sharif, M. and Ahmad, Z.: Acta Physica Polonica B 39(2008)1337.

[15] Israel, W.: Nuovo Cimento B44(1966)1.

[16] Israel, W.: Nuovo Cimento B48(1967)463(E).

[17] Lemos, J.P.S.: Phys. Rev. D57(1998)4600.

[18] Cai, R.G. and Wang, A.: Phys. Rev. D73(2006)063005.

[19] Herrera, L. and Santos, N.O.: Phys. Rev. D70(2004)084004.

[20] Herrera, L. and Santos, N.O.: Class. Quantum Grav.22(2005)2407.

[21] Villas de Rocha, J.F., Wang, A. and Santos, N.O.: Phys. Lett. A255(1999)213.

[22] Pereira, P.R.C.T. and Wang, A.: Phys. Rev. D62(2000)124001.

[23] Pereira, P.R.C.T. and Wang, A.: Phys. Rev. D67(2003)129902(E).

[24] Sharif, M and Ahmad, Z.: Int. J. Mod. Phys. A23(2008)181.

[25] Santos, N.O.: Phys. Lett. A106(1984)296.

[26] Santos, N.O.: Mon. Not. R. Astr. Soc. 216(1985)403.

[27] Hawking, S.W. and Ellis, G.F.R: The Large Scale Structure of Spacetime (Cambridge University Press, Cambridge, 1973).

[28] Qadir, A.: J. Math. Phys. 33(1992)6. 